\title{
Caracterización de los Estilos de Aprendizaje de estudiantes de básica primaria de un grupo étnico*
}

\author{
[Versión en Castellano] \\ Characterization of the Learning Styles of Elementary School \\ Students from an Ethnic Group
}

Caracterização dos Estilos de Aprendizagem de alunos do Ensino Fundamental de um grupo étnico

Recibido el 19 de junio, 2019. Aceptado el 24 de junio, 2020.

\author{
María-Disney Palacios-Rentería** \\ https://orcid.org/0000-0002-7698-067X
}

Colombia

- Para citar este artículo:

Palacios-Rentería, María-Disney

\section{Resumen}

(2021). Caracterización de

los Estilos de Aprendizaje de estudiantes de básica primaria de un grupo étnico.

Ánfora, 28(50), 249-274.

https://doi.org/10.30854/anfv28.n50.2021.787

Universidad Autónoma de

Manizales. ISSN 0121-6538/

e-ISSN 2248-6941.

CC BY-NC-SA 4.0

Objetivo: caracterizar los estilos de aprendizaje de estudiantes de básica primaria del grupo étnico Emberá Chamí del corregimiento de Caimalito, Pereira. Metodología: se optó por un enfoque cuantitativo con diseño transversal descriptivo. El recabado de la información se realizó a través del cuestionario CHAEAJunior. Resultados: se evidenció una preferencia en los estilos reflexivo y pragmático al analizar de manera discriminada los estilos de aprendizaje. Sin embargo, al someter los datos a una prueba de hipótesis se

\footnotetext{
* Este artículo deriva de la investigación "Caracterización de los estilos de aprendizaje de estudiantes de básica primaria de un grupo étnico", realizado en el marco de la Maestría en desarrollo infantil de la Universidad de Manizales. La investigación forma parte del macroproyecto Estilos cognitivos, autoconcepto y autorregulación para el aprendizaje en niños y niñas escolarizados de diferentes municipios del país, que se realizó entre 2017-2019. Con identificador http://ridum.umanizales.edu.co:8080/xmlui/handle/6789/3964. La autora declara que no hubo conflicto de intereses en la ejecución del proyecto de investigación.

** Magíster en Desarrollo Infantil. Licenciada en Educación básica. Docente de la Institución Educativa Gabriel Trujillo del municipio de Pereira, Risaralda, Colombia. Correo electrónico: disneipa85@hotmail.es; paremadis@gmail.com
} 
encontró que no existen diferencias significativas en los estilos de aprendizaje entre los participantes del grupo Emberá. Conclusiones: la caracterización de los estilos de aprendizaje en la población étnica objeto de estudio presenta rasgos similares a los hallados en otras investigaciones, en las que también se concluye una tendencia reiterada por los estilos reflexivo y pragmático.

Palabras-clave: Estilos de aprendizaje; Proceso de aprendizaje; Grupo étnico; Emberá Chamí; Estudiantes básica primaria.

\section{Abstract}

Objective: to characterize the learning styles of elementary school students from the Emberá Chamí ethnic group from the town of Caimalito, Pereira. Methodology: a quantitative approach with a descriptive cross-sectional design was chosen. The information was collected through the CHAEA-Junior questionnaire. Results: a preference in reflective and pragmatic styles was evidenced when analyzing learning styles in a discriminatory way. However, when submitting the data to a hypothesis test it was found that there are no significant differences in learning styles among the participants of the Emberá group. Conclusions: the characterization of learning styles in the ethnic population under study presents features similar to those found in other investigations, in which a reiterated trend towards reflective and pragmatic styles is also concluded.

Keywords: Learning styles; Learning process; Ethnic group; Embera Chamí; Basic elementary students. 


\section{Resumo}

Objetivo: caracterizar os estilos de aprendizagem de alunos do ensino fundamental da etnia Emberá Chamí do município de Caimalito, Pereira. Metodologia: optou-se por uma abordagem quantitativa com um desenho transversal descritivo. As informações foram coletadas por meio do questionário CHAEA-Junior. Resultados: foi evidenciada uma preferência pelos estilos reflexivo e pragmático ao analisar estilos de aprendizagem de forma discriminatória. Porém, ao submeter os dados a um teste de hipóteses, constatou-se que não existem diferenças significativas nos estilos de aprendizagem entre os participantes do grupo Emberá. Conclusões: a caracterização dos estilos de aprendizagem na população étnica em estudo apresenta características semelhantes às encontradas em outras investigações, nas quais também se conclui uma tendência reiterada aos estilos reflexivo e pragmático.

Palabras-chave: Estilos de aprendizagem; Processo de aprendizagem; Grupo étnico; Emberá Chamí; Alunos do ensino fundamental básico. 


\section{Introducción}

En el marco de la comprensión actual sobre el concepto de diversidad existe una incoherencia entre el uso normativo y el uso práctico del concepto. Por ello, la respuesta a la diversidad es, tal vez, uno de los retos importantes y difíciles que enfrentan los equipos docentes; a ello se suma la ausencia de esfuerzos de algunos de estos actores para reconocer la diversidad de estudiantes en el aula.

Lo anterior lleva a inferir que el problema no es la diversidad sino que, aunque se tiene el conocimiento y está incorporado en el docente que se es, en muchas ocasiones es el sedentarismo práctico y el desconocimiento sobre el estudio de la diversidad. Para Devalle y Vega (2006), "el problema es la forma cómo se concibe y se pone en práctica la valorización de las diferencias" (p. 14). De este modo, comprender la diversidad es reconocer que todos son diferentes y que tales diferencias refieren formas de sentir, pensar, interpretar y representar el mundo, así como de aprender e interactuar en los ambientes de aprendizaje.

De acuerdo con estas autoras, no es suficiente la existencia de un currículo diferenciado, pues de nada sirve un currículo con el que se pretenda incluir y reconocer todos los estilos de aprendizaje, formas de ser y hacer en el interior del aula, si se sigue tratando a los estudiantes como si aprendieran del mismo modo; de tal manera que si el docente no comprende desde la acción, no podrá hacer frente a la diversidad de estilos y ritmos de aprendizaje que sus estudiantes presentan y, por lo tanto, no estará en la capacidad de emplear nuevas estrategias de enseñanza que potencien, mejoren y aprovechen de un modo adecuado la diversidad cognitiva y étnica que hay en el aula.

Ahora, la diversidad otorga un carácter singular, único e irrepetible, propio a cada sujeto y en el que juega un papel importante la escuela como espacio de reconocimiento de los ritmos, estilos, formas de pensar y rasgos de personalidad. En este contexto, el docente tiene como misión no solo reconocer estas diferencias, sino que debe potenciarlas, para optimizar los procesos de aprendizaje y las estrategias de enseñanza apropiadas desde la comprensión de los estilos particulares de sus estudiantes como rasgos distintivos y característicos que pueden ayudar a mejorar su proceso formativo.

Desde el punto de vista de Hederich (2013), los estilos constituyen "una expresión de la personalidad consistente en una disposición al uso de ciertas habilidades cognitivas" (p. 25); así, se podría afirmar que la estilística es un rasgo característico y diferenciador, en tanto funciona como "una manera particular de tipificar la conducta individual” (Hederich, 2013, p. 25). En este sentido, según lo planteado por Lozano (2006), los estilos son un conjunto de preferencias, tendencias y disposiciones que tiene una persona para hacer algo, y estos se mani- 
fiestan por medio de lo conductual y de diversas fortalezas que distinguen a un individuo de los demás.

Asimismo, en el ámbito educativo, "la estilística se refiriere a la identificación, apreciación y clasificación de las diversas formas de aprender y enseñar" (Lozano, 2006; citado en Hederich y Camargo, 2015, p. 136). Desde este ámbito, por su aplicabilidad se encuentran los estilos cognitivos con autores como Messick (2010); los estilos de enseñanza con González-Peiteado (2013) e Isaza y Henao (2012); también están los estilos de pensamientos, los estilos pedagógicos, los de comunicación, los de liderazgo y los de aprendizaje.

En torno a los estilos de aprendizaje, Curry (1987), a través del modelo de la cebolla, como se le conoce en habla hispana, clasificó los modelos de estilo de aprendizaje al diferenciar tres capas. La primera capa, la parte exterior de la cebolla, la más fácil de observar, en la que están las preferencias instruccionales y ambientales de aprendizaje, es decir, las estrategias de aprendizaje implementadas por el estudiante; en la segunda capa, se ubican las preferencias acerca de cómo se procesa la información -en este caso, las preferencias son los estilos de aprendizaje-; finalmente, la tercera capa, la más interna, se relaciona con las preferencias de aprendizaje de acuerdo con la personalidad, esto es los estilos cognitivos (Santaolalla, Gallego y Urosa, 2015).

A partir de lo expuesto, los estilos de aprendizaje pueden definirse como "la predisposición por parte de los estudiantes para adoptar una estrategia de aprendizaje particular independiente de las demandas específicas de la tarea” (Schmeck, 1983; citado en Uva, 2017). De acuerdo con Keefe, 1988; citado en Terrádez, s. f.), los estilos de aprendizaje constituyen un conglomerado de rasgos afectivos, cognitivos y fisiológicos, los cuales se convierten en indicadores estables para reconocer cómo percibe, interactúa y responde el estudiante al ambiente de aprendizaje.

En este sentido, los estilos de aprendizaje se hacen visibles en el momento en que el estudiante se enfrenta a la tarea de aprender. De tal modo que los estilos de aprendizaje se comprenden como la forma ideal y preferente que un estudiante tiene y manifiesta, consciente o inconscientemente, al momento de ejecutar o llevar a cabo una demanda de aprendizaje. Evidencia de esto se encuentra en los estudios realizados González-Peiteado (2013); Chiang, Díaz y Arriagada (2016); Rojas, Zarate y Lozano (2016); Pantoja, Duque y Meneses (2013).

De otro lado, en Chile está la investigación realizada por Cáceres y Vilchez (2012): "Chaea Junior ${ }^{1}$ en estudiantes de la comuna de Talcahuano", en la que

1. El cuestionario sobre estilos de aprendizaje CHAEA-Junior es de alta difusión y aplicación. Su constructo se basa en la teoría del ciclo de aprendizaje basado en la experiencia de David Kolb, en tanto registra la manera como un estudiante se ajusta, de acuerdo con su experiencia de aprendizaje, a una determina preferencia estilística. En ese contexto, debido a que la población en la que se implementó se encuentra 
participaron 1700 estudiantes de los grados cuarto a octavo, de cinco centros educativos de Educación General Básica (EGB), cuyas edades fluctuaban entre los 9 y 15 años de edad; tal estudio se llevó a cabo con la aplicación del cuestionario de Sotillo (2014).

En Brasil, la investigación de Coehlo et al. (2013), "Análisis entre la asociación de los estilos de aprendizaje con el género, banda etaria e inteligencia de los niños brasileños de la enseñanza fundamental”, contó con una muestra de 49 niños y niñas de básica primaria; la investigación tenía el propósito de analizar la relación entre los estilos de aprendizaje, el género, la edad y la inteligencia, utilizando el instrumento HAQLS, adaptación del CHAEA por Portillo, y traducido al portugués con 12 preguntas, cuatro respuestas y el IQ para la inteligencia.

Los resultados, en este caso, mostraron que los estilos predominantes fueron el reflexivo y el pragmático, y con menor influencia el activo y el teórico; en cuanto al sexo, los niños reflejaron el estilo activo y las niñas el estilo pragmático y no se encontraron diferencias entre el estilo y la inteligencia.

En México, el estudio realizado por Mejía y Jaik (2014), "Estilos de aprendizaje de docentes y alumnos y su relación con el rendimiento académico en educación primaria", tuvo la participación de 145 alumnos de los grados cuarto a sexto de básica primaria de tres instituciones educativas, con edades comprendidas entre los 9 y 14 años (53 mujeres y 47 hombres), y la utilización del cuestionario CIEA, elaborado a partir del cuestionario CHAEA de Honey y Alonso (2002). Allí se dedujo que los hombres tienen una mayor preferencia por el estilo reflexivo y pragmático que las mujeres; también se evidenció que a mayor edad prevalecen los estilos reflexivos y teóricos, y que el grado escolar no determina la preferencia estilística. En general, el estudio mostró que los alumnos en su mayoría presentan el estilo reflexivo.

En el caso de Perú, el trabajo de Díaz (2017), "Estilos de aprendizaje en estudiantes de educación primaria en el distrito de Sapallanga", tuvo la participación de 76 estudiantes, en su mayoría representados por mujeres (40) con un $53 \%$ y en menor cantidad con hombres (36) con $47 \%$, cuyas edades fluctuaban entre los 8 y 14 años, de los cuales 37 pertenecían al grado cuarto y 39 al grado quinto. Los resultados de la investigación indicaron que en el grado cuarto la mayoría presentan estilo auditivo (conformado por 17 estudiantes), 13 son visuales y 7 kinestésicos, mientras que en quinto grado la mayoría son de estilo

escolarizada, según los lineamientos del MEN (Ministerio de Educación Nacional), se estima que su utilización es legítima, dado que se trata de caracterizar la experiencia y derivar de dicha experiencia una preferencia hacia el aprendizaje, la cual puede ser reportada por cualquier sujeto independientemente de su etnicidad, raza o credo. 
visual, con un total de 17 estudiantes, y los demás presentaron estilo auditivo y kinestésico con igual porcentaje.

En relación con los dos grados, se observó que la mayoría, es decir, el 39\% de estudiantes tienen el estilo visual, el $36 \%$ cuentan con el estilo auditivo y el $23 \%$ con el aprendizaje kinestésico; por consiguiente, se encontró que 15 estudiantes mujeres mantenían un estilo visual, 16 el auditivo y 9 el kinestésico, y en cuanto a los hombres, se halló que 15 tenían estilo visual, 12 auditivo y 9 kinestésico (Díaz, 2017).

Igualmente, Granados y García (2016) demostraron que la gran mayoría de los estudiantes tienen marcadas preferencias por el estilo reflexivo, con un 29.6\%, seguido del estilo teórico con 25.7\%.

En España, un estudio de Sotillo (2014) concluyó que, en su mayoría, los estudiantes son reflexivos mientras que el resto muestra una combinación de los estilos teóricos, activo y reflexivo.

En Colombia, un estudio de Ortiz (2013) arrojó que un $8 \%$ tienen estilo auditivo, otro $8 \%$ estilo visual y el $17 \%$ tiene un estilo kinestésico. En cuanto al sexo, se observó que un 16\% tienen estilo kinestésico y tanto las mujeres los hombres prefieren un estilo multimodal.

Por otra parte, la tesis de maestría realizada por Herrera (2014) en Armenia-Colombia revela que las instituciones de las zonas rurales no reflejan un perfil estilístico definido; además, los estilos que más predominaron fueron el convergente Kolb $(1984,1985)$, que indica que en las mujeres su preferencia estilística estuvo más inclinada hacia el estilo activo y teórico y, en los hombres, hacia el estilo reflexivo, teórico y pragmático.

Igualmente, en el trabajo de García y Sáchica (2016), demostraron que la gran mayoría de los estudiantes tiene marcadas preferencias por el estilo reflexivo, seguido del estilo teórico, mientras que los estilos activos y pragmáticos presentaban preferencias bajas.

En el estudio de Cánchala, Bastidas y Velásquez (2019), "Estilos de aprendizaje en escolares indígenas del Gran Mallama”, revelaron que el estilo predominante fue el auditivo y el canal con menor preferencia fue el visual. Una de las conclusiones es que la mayoría de los estudiantes tienen como estilo principal el auditivo y, en este caso, el mayor porcentaje se observó en las niñas.

En el municipio de soledad departamento de Atlántico, Colombia, la investigación de Noriega y Sánchez (2019) demostró que existe una correlación directa entre los estilos de aprendizaje y los procesos de evaluación que aplican los docentes en el aula. Se notó que en los grados terceros, cuartos y quinto prevalece el estilo experimentación activa y, respecto al estilo experiencia concreta, no se encontró preferencia estilística. León (2015), por su parte, en su estudio reveló 
que el estilo reflexivo le posibilita al estudiante obtener un mayor rendimiento académico.

En consecuencia, parecen existir dos factores primordiales en el estudio estilístico del estudiante. El primero, la mayoría de estudios parecen coincidir en que el estilo reflexivo es el más recurrente, lo que claramente causa cierta curiosidad, pues es paradójico que el estudio al realizarse sobre población hispanoamericana de países distintos y muestras diversas arroje resultados similares sobre la preferencia hacia un estilo de aprendizaje específico. De otro lado, la ausencia o desconocimiento de los docentes para incluir dentro de sus prácticas el concepto de diversidad e inclusión, podría constituir una posible explicación del primer factor.

Es decir, que no exista un modelo educativo que privilegia y potencia las diferencias individuales de aprendizaje lleva a la prevalencia de un estilo de aprendizaje, el cual podría corresponder al estilo del docente de aula y no, necesariamente, el estilo del estudiante; esta es la problemática que, probablemente, esté generando bajo rendimiento académico, repitencia escolar, sobre todo, en la mayoría en condición extra edad. Estos parecen ser los factores que causan desmotivación frente al proceso educativo y, por consiguiente, las continuas inasistencias a clase.

Esta problemática en conjunto con los escasos ingresos económicos, tal vez son las causas que han motivado a los padres y/o acudientes a tomar la decisión de introducir a la población masculina desde los 13 años a laborar cogiendo café y a la población femenina casarlas cuando cumplen los 15 años; todo esto, si no avanzan en los procesos académicos.

Por todo lo anterior, el presente artículo de investigación se propone abordar la caracterización de los estilos de aprendizaje de un grupo étnico de niños Emberá; estudiar los estilos de aprendizaje en este grupo poblacional lleva a identificar las preferencias estilísticas de los educandos, con el fin de generar insumo teórico-práctico que permita que los docentes puedan aplicar un trato específico y diferencial a cada estudiante dependiendo de su estilo; también se podría aportar que los alumnos conozcan sus estilos y se motiven a utilizar otros, procurando que los docentes adopten estrategias pedagógicas adecuadas para obtener una labor educativa eficaz y eficiente que contrarreste el alto índice de mortalidad y repitencia académica, desescolarización, población con extra edad, deserción y desmotivación de los padres y/o acudientes, promoviendo una mirada diferencial frente a la educación.

Un insumo teórico-práctico que al mismo tiempo que contribuya a la formación de sujetos integrales, que interactúen con el/los otro/s, propiciando un buen intercambio de saberes y mayor participación, en el que se fomenten dis- 
tintos valores logrando así una sana convivencia basada en el respeto y la aceptación del Otro.

Así pues, el presente trabajo busca caracterizar los estilos de aprendizaje de estudiantes de básica primaria de un grupo étnico según la edad y el sexo, en una institución educativa del corregimiento de Caimalito, Pereira (Colombia). Se espera corroborar si existe o no preferencia estilística en los niños Emberá o si esta preferencia, en realidad es producto de su adaptación a un determinado contexto educativo en el cual se siguen modelos educativos estandarizados.

\section{Metodología}

La presente investigación se abordó desde un estudio empírico analítico con enfoque cuantitativo y con diseño transversal descriptivo que se comprende como el tipo de diseño en el que se recogen los datos en un único momento y, a partir de los mismos, se especifican, de manera descriptiva, propiedades y características importantes de una determinada población Hernández, Fernández y Baptista (2014).

El estudio se realizó en tres momentos. En un primer momento, se hizo una prueba piloto, con el fin de observar la pertinencia del instrumento para este grupo étnico, de acuerdo con el grado de complejidad lingüística, en cuanto al léxico se refiere. Posteriormente, se aplicó el cuestionario CHAEA-Junior, en su versión corta adaptada por Sotillo (2014). En la tercera fase, se elaboró la base de datos (organización de variables en Excel para su organización, depuración y codificación) y, luego, se procedió a su análisis, a través del Software IBM SPSS versión 22.

\section{Muestra}

La población estuvo comprendida por $(\mathrm{N}=23)$ estudiantes de la comunidad Emberá con una edad promedio observada de $(\mathrm{M}=11,2$ años, $\mathrm{DE}=2,059)$. En los participantes prevaleció el sexo masculino $(65,2 \%)$ sobre el sexo femenino (34.7\%). Los participantes pertenecen a una institución educativa del corregimiento de Caimalito del municipio de Pereira (Risaralda, Colombia) del resguardo indígena Emberá Chamí de los grados cuarto y quinto de básica primaria. En cuanto a las condiciones sociales, muchos de los estudiantes presentan condiciones de vulnerabilidad y son provenientes de los municipios con altos niveles de desplazamiento.

Esta comunidad étnica depende de la agricultura y la artesanía como actividades económicas primarias. La estructura de la familia es extensa, en su mayo- 
ría pueden vivir alrededor de cuatro familias en una sola vivienda. A su vez, para aprender a leer y a escribir en su lengua nativa "Emberá" lo realizan por medio de la creencia "jiru pota war" (el niño nacido de la pierna). Sus mitos relatan no solo el origen del mundo, de los animales, de las plantas y del hombre, sino todos los acontecimientos que hacen al hombre de este tiempo. La creencia "jiru pota war", junto con el origen del agua, es considerada fundamental para su identidad.

Cabe aclarar, que si bien el cuestionario CHAEA-Junior no fue pensado para la caracterización de grupos étnicos, al estar éstos niños inmersos en la cultura de habla hispana y hacer parte de procesos de formación promovidos por el Estado, el vocabulario así, como su uso contextual, no representó una dificultad adicional para su lectura, interpretación y comprensión para los participantes.

\section{Instrumentos de recolección de información}

Se utilizó como instrumento único de recolección de la información el Cuestionario CHAEA-Junior de Estilos de Aprendizaje (anexo 1) de HoneyAlonso (2002), en la versión corta adaptada por Sotillo (2014) para poblaciones de primaria.

\section{Caracterización del instrumento}

El CHAEA-Junior es el resultado de la investigación realizada por Sotillo (2014): "Los Estilos de Aprendizaje en alumnos de Primaria: Diagnóstico y propuesta pedagógica”. El CHAEA-Junior fue adaptado para estudiantes de $4^{\circ}$ y $5^{\circ}$ Primaria; no obstante, se vio también su idoneidad, a lo largo de la investigación, para los alumnos del Primer Ciclo de Secundaria Obligatoria, es decir, grados $6^{\circ}$ y $7^{\circ}$. El CHAEA-Junior está conformado por (44) ítems los cuales contienen afirmaciones acerca de la preferencia por situaciones de aprendizaje o gusto personal. Las respuestas se obtienen a través de una escala nominal dicotómica donde el estudiante debe marcar el signo $(+)$ si está de acuerdo y el signo menos (-) si no está de acuerdo. La versión del CHAEA-Junior presentada por Sotillo (2014) no requiere del baremo para hallar el estilo diferente, obteniéndose éste por la suma de puntajes directos.

$\mathrm{El}$ instrumento presenta un lenguaje sencillo con términos coloquiales para que pueda ser comprendido y leído por niños entre edades de 9 a 14 años, desde un punto de vista sintáctico y semántico. La distribución de los ítems es aleatoria y se subdivide en cuatro grupos de 11 ítems, a través de los cuales resulta posible caracterizar los cuatro estilos de aprendizaje: Activo, Reflexivo, Teórico y Pragmático (Granados y García, 2016). 
La puntuación absoluta se obtiene, según cada estilo, con un único valor de rango de (0 a 11), obtenido de la suma directa de la respuesta. El valor que resulte mayor será la preferencia dominante y el que resulte superior después de este es el siguiente estilo que predomina. Conviene agregar que para validar el cuestionario, se recurrió a un tribunal de 5 expertos (las doctoras: Daniela Melaré, Mari Luz Cacheiro, María Isabel Adán León y los doctores: Pedro Martín Geijo y José Luis García Cué), investigadores de gran experiencia en los Estilos de Aprendizaje.

Este cuestionario ha sido validado en Colombia por los siguientes investigadores: Varela (2014) de Antioquia; Gómez, Jaimes y Sereviche (2017) de Cartagena; Acevedo, Cavadia y Alvis (2015) de Cartagena; Ángel y Alonso (2012) de Bogotá; Casadiego (2015) en el Cesar; Mendoza (2012) en Cali y, en Manizales, por Granados y García (2016).

Los resultados de la caracterización del grupo étnico se presentarán de manera discriminada por cada estilo. Esto, con el fin de identificar a los participantes de manera detallada en relación a cada uno de los estudios de aprendizaje propuestos por Honey y Alonso (2002). Posterior a esta discriminación por cada uno de los estilos, se muestra la caracterización del comportamiento estilístico de los participantes. Finalmente, se cerrará con una prueba para determinar si existen o no diferencias significativamente estadísticas entre cada uno de los perfiles estilísticos encontrados y poder establecer así, si es posible hablar de una estilística preferente en el aprendizaje de los niños Emberá o si, por el contrario, no se observa una preferencia en particular.

\section{Resultados}

\section{Caracterización por estilo de aprendizaje de acuerdo con la preferencia alta/baja}

Se encontró, como lo muestra la figura 1, una pequeña divergencia entre la preferencia por el estilo activo en los participantes. De acuerdo con los resultados se observó una diferencia entre preferencia alta y baja del 13\%, lo que permite deducir que no existe una tendencia de los participantes hacia este estilo. Los resultados se muestran en la figura 1. 
Figura 1. Distribución de estudiantes para el estilo activo

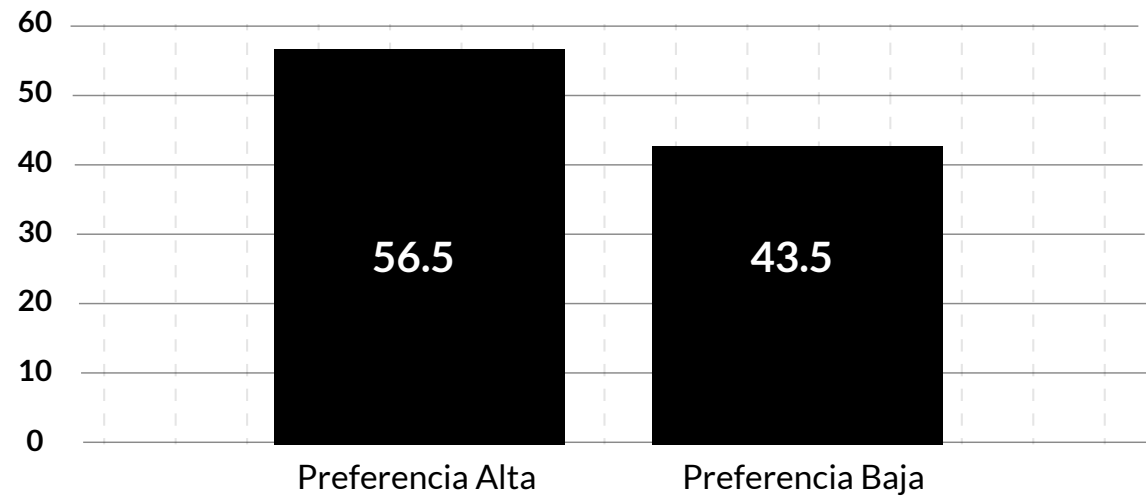

Fuente: elaboración propia

En cuanto al estilo de aprendizaje reflexivo se encontró una diferencia del $47,8 \%$ entre los participantes en relación con la preferencia alta/baja. El elevado valor de diferencia indica una posible tendencia o preferencia de los participantes hacia este estilo de aprendizaje (figura 2):

Figura 2. Distribución de estudiantes para el estilo reflexivo

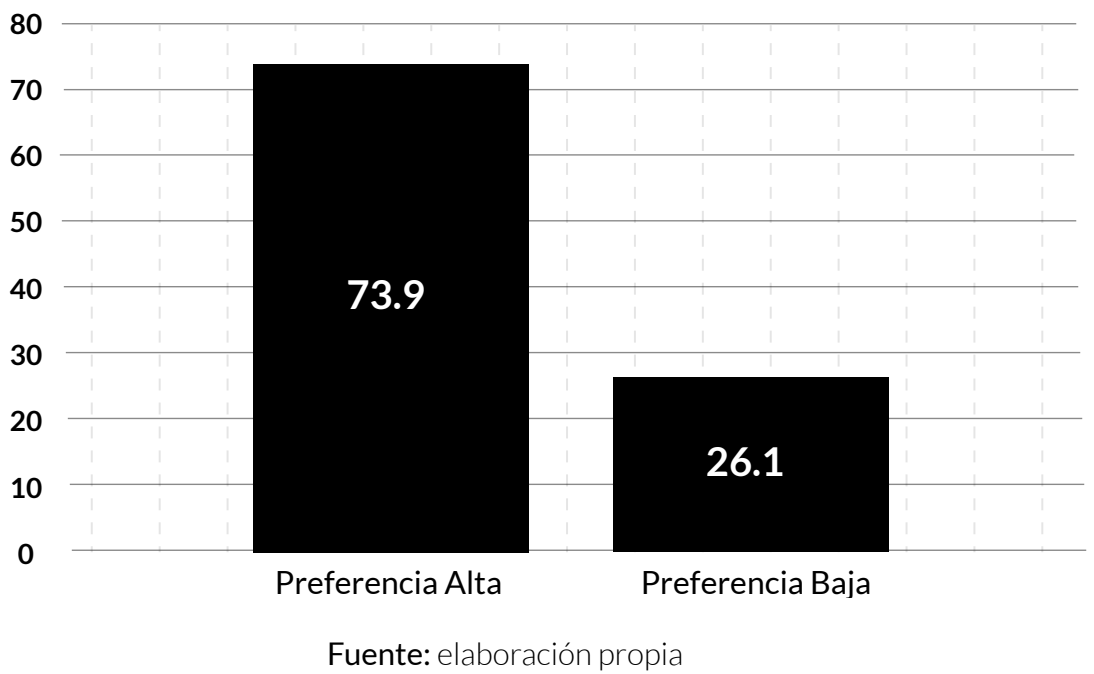


En el estilo teórico, se observó una diferencia de 21,8\% en relación con la preferencia estilística alta/baja. De acuerdo con este resultado, se puede estimar que no existe una diferencia marcada entre la población en relación para el estilo de aprendizaje teórico (figura 3).

Figura 3. Distribución de estudiantes para el estilo teórico

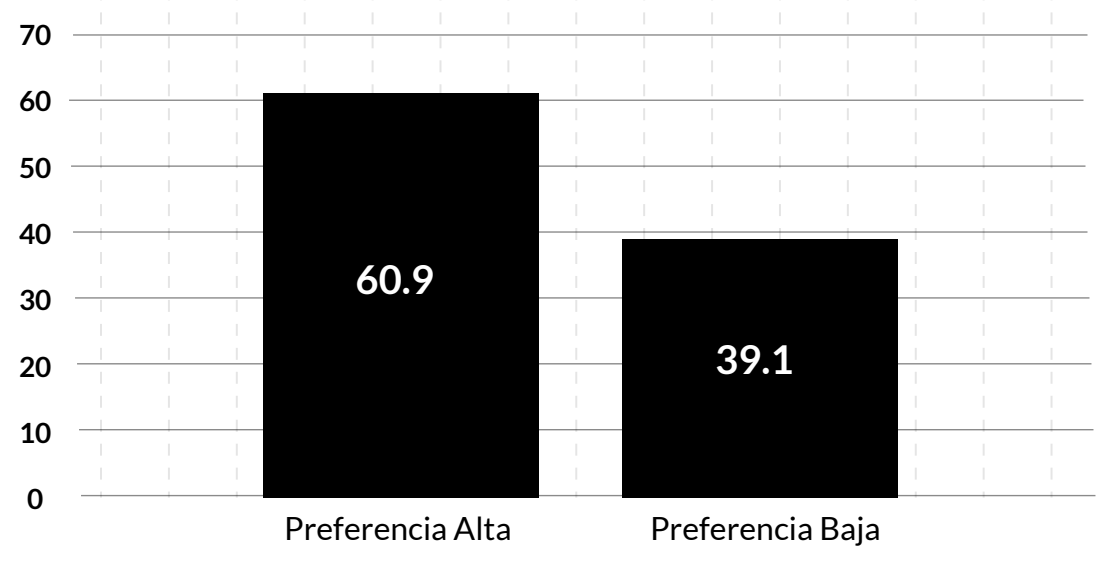

Fuente: elaboración propia

Finalmente, los resultados para la preferencia estilística pragmática alta/ bajo arrojaron una diferencia del 30,4\% entre los participantes; esto lleva a considerar que este podría constituir el segundo rasgo estilístico de la comunidad étnica participante (figura 4): 
Figura 4. Distribución de estudiantes para el estilo Pragmático

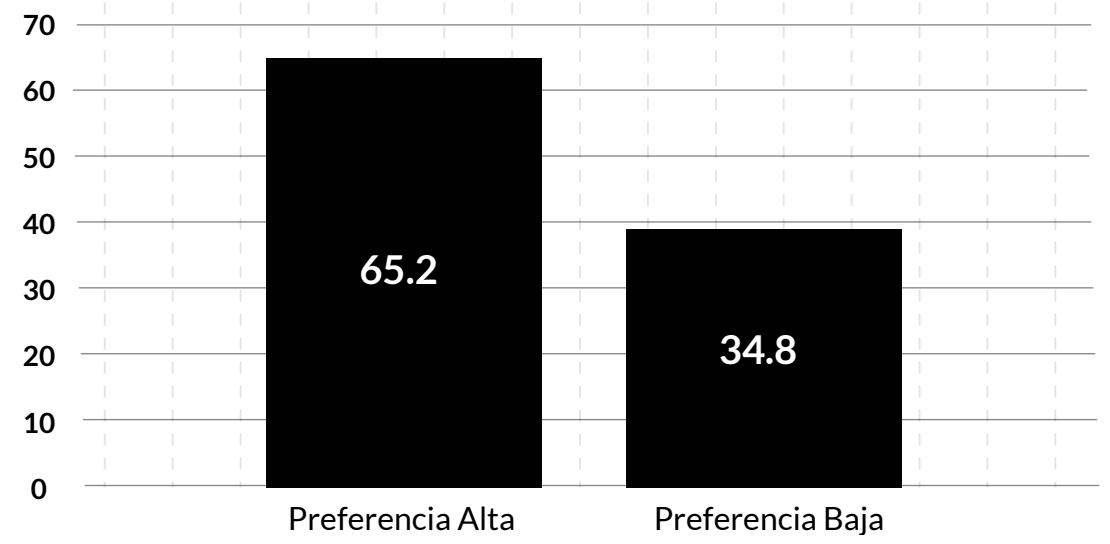

Fuente: elaboración propia

\section{Caracterización general de las preferencias estilísticas de aprendizaje alta/baja}

Los resultados generales en relación con la preferencia estilística permiten observar de manera más clara una tendencia de los participantes hacia los estilos reflexivos $(73,9 \%)$ y pragmático $(65,2 \%)$. Sin embargo, en términos generales no se observan grandes diferencias entre los perfiles estilísticos, aunque sí se podría plantear cierta tendencia de acuerdo con los resultados (figura 5). 
Figura 5. Distribución de estudiantes para el estilo pragmático

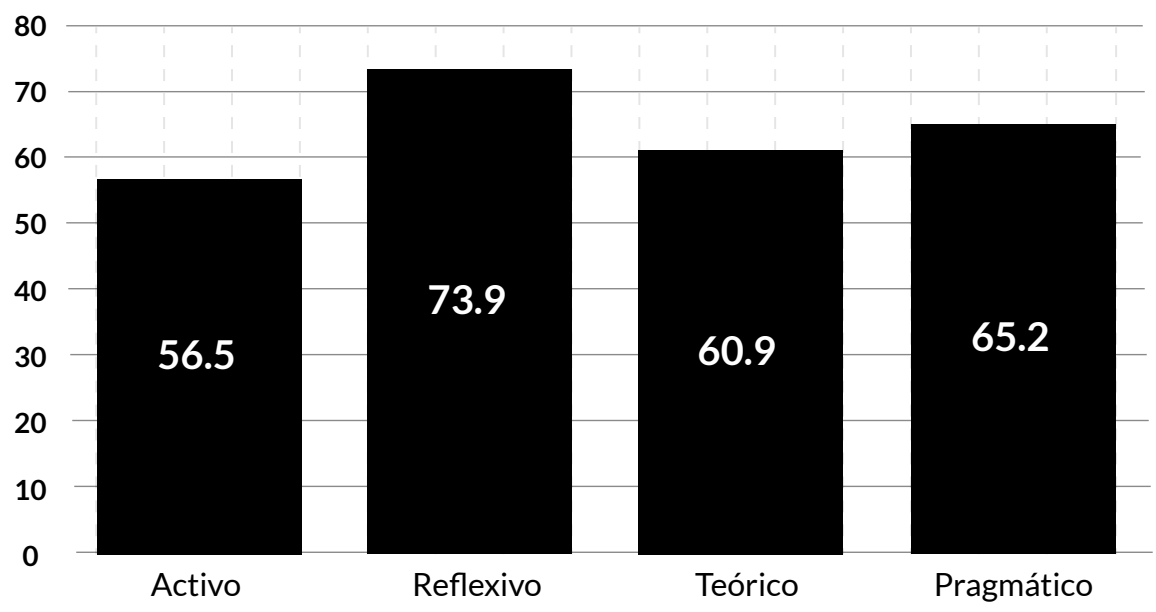

Fuente: elaboración propia

Si bien los resultados sobre la descripción de cada uno de los estilos de aprendizaje de manera discriminada y general, permiten ver lo que podría constituir un perfil estilístico de los niños del grupo étnico centrados en los estilos reflexivo y pragmático, se quiso corroborar si existían o no diferencias significativas entre los estilos de aprendizaje reportados por los estudiantes. Para realizar la prueba se optó por la formulación de una hipótesis la cual, consistió en indagar por la existencia de un perfil estilístico en el grupo étnico de niños participantes.

La hipótesis formulada fue:

$\mathrm{H}^{0} \mathrm{El}$ grupo de participantes no presenta una preferencia diferente en sus estilos de aprendizaje.

$\mathrm{H}^{1} \mathrm{El}$ grupo de participantes presenta una preferencia diferente en sus estilos de aprendizaje.

Para llevar a cabo la testación de la hipótesis y al tratarse de un análisis en el que se incluyen más de dos variables, se optó por la prueba de Kruskal-Wallis. Esta prueba se utiliza cuando se desea testar si varias muestras $(\mathrm{K})$ provienen o no de una misma población. Para este caso, si los estilos de aprendizaje son o no iguales para todo el grupo étnico de niños participantes. 
Los resultados en la prueba de hipótesis a través del método no paramétrico de KS muestras, arrojó un $P$ valor mayor a $(\mathrm{P}>0.05)$ para cada uno de los estilos de aprendizaje; esto implica la aceptación de la hipótesis nula. Es decir, que no existe una diferencia entre la preferencia de estilos de aprendizaje para el grupo étnico participante en los resultados del presente estudio. Los resultados de la prueba KS-muestras para el estilo activo arrojó un valor de (,469), para el estilo reflexivo de (,734), para el estilo teórico de (,644) y para el estilo pragmático de (,618). Estos resultados permiten advertir que ningún estilo resulta en particular preferente para el grupo étnico participante en particular (tabla 1):

Tabla 1. Estilos de aprendizaje según la edad

\begin{tabular}{c|c|c|c|c|}
\hline \multicolumn{5}{|c|}{ Estadísticos de prueba ${ }^{\mathbf{a}, \mathrm{b}}$} \\
\hline & Activo & Reflexivo & Teórico & Pragmático \\
\hline Chi-cuadrado & 6.625 & 4.389 & 5.133 & 5.344 \\
\hline Gl & 7 & 7 & 7 & 7 \\
\hline $\begin{array}{c}\text { Significancia asin- } \\
\text { tótica }\end{array}$ & .469 & .734 & .644 & .618 \\
\hline \multicolumn{5}{|c|}{ a. Prueba de Kruskal-Wallis } \\
\hline \multicolumn{5}{|c|}{ b. Variable de agrupación: edad } \\
\hline
\end{tabular}

Fuente: elaboración propia

\section{Discusiones}

Si bien en la prueba de hipótesis se negó la posibilidad de encontrar una preferencia estilística en el grupo étnico de niños Embera Chamí de acuerdo con los resultados de la prueba KS-muestras, la discusión se realizará en relación a los dos estilos más frecuentes de preferencia alta observados (Reflexivo 73,9\% y Pragmático 65,2\%). Estos resultados, aunque no permiten diagnosticar que constituyen un estilo de aprendizaje propio para la comunidad de niños Emberá participantes, sí pueden generan una comprensión del impacto que ha tenido en ellos el proceso de educación estatal, lo cual permite que sea comparable con otras investigaciones en las que se han caracterizado los estilos de aprendizaje en estudiantes de centros educativos públicos.

Es así como los resultados de este trabajo son contrastables con los hallazgos de Coehlo et al. (2013) en una investigación llevada a cabo en Brasil en la que encontró una predominancia de los estilos reflexivo y pragmático y una menor influencia de los estilos activo y teórico. De igual manera, en su investigación 
Cáceres y Vilchez (2012), en Chile, aplicaron el mismo cuestionario y observaron una preferencia estilística por el estilo reflexivo (34\%) y del pragmático (10\%).

En el mismo sentido, los resultados parciales del presente artículo se asemejan a los obtenidos por Mejía y Jaik (2014) en México, quienes encontraron que los alumnos -en su mayoría-presentan una preferencia estilística del tipo reflexiva. De igual manera, en la investigación realizada por Herrera (2014) en Armenia (Colombia) se aplicó el instrumento de Kolb y se obtuvo una marcada tendencia de la población participante por el estilo reflexivo.

En la investigación de Granados y García (2016) se reportaron resultados similares a los hallados aquí al coincidir que el estilo reflexivo $(29,6 \%)$ es uno de los más frecuentes en los estudiantes de primaria de grados $4^{\circ}$ y $5^{\circ}$ reflexivo. Así mismo, los resultados parecen similares a los obtenidos por León (2015) en Colombia, quien evidenció una predominancia del estilo reflexivo (53 \%) sobre el resto de estilos de aprendizaje.

De igual manera, los resultados de Rodríguez (2014) plantean que el estilo reflexivo, por lo general, puntúa entre los estilos de aprendizaje más frecuentes al realizar la caracterización de la estilística de aprendizaje. En el mismo sentido, Chiang et al. (2013) encontró que el estilo reflexivo está asociado con el tipo de estudiantes que dedican mayor tiempo a pensar en cómo resolver un problema de aprendizaje o ejecutar una tarea.

Por su parte, Ferrés-Gurt, Marbà-Tallada y Sanmartí-Puig (2014) encontraron que el estilo reflexivo parece asociarse con estudiantes cuya abstracción y sobre carga inductiva generan pesos negativos en sus desempeños académicos.

Sotillo (2014) plantea, que los estilos de mayor recurrencia en los estudiantes de primaria pareciesen centrarse en los estilos reflexivos y teóricos. Estos aspectos que podrían confirmar que quizá no se trata de un auténtico estilo de aprendizaje en los estudiantes, sino de cierta disposición del sistema escolar en el que se encuentran, y tal sistema se ha derivado de una tradición eminentemente enciclopédica que sigue privilegiando la memoria; de ahí el rasgo teórico y la reflexión como mecanismo base de la enseñanza.

\section{Conclusiones}

Se concluye que el grupo étnico de niños Embera-Chamí presenta una tendencia hacia los estilos reflexivos y pragmáticos; dicha preferencia parece ser fruto del sistema escolar en el que se encuentran y en el cual no existe una diferenciación ni preferencia étnica en términos de procurar el desarrollo de las facultades cognitivas y estilísticas de los estudiantes. 
En las aulas ocurren altas concentraciones de niños de comunidades -incluso sin ninguna pertenencia a una etnia específica- en clases magistrales en las que obedecen guiados por las demandas de docentes que imparten las clases de manera general y sin un reconocimiento positivo de la diversidad o inclusión. Este factor es reflejo del modelo de educación estatal que hace parte de reformas educativas lideradas por organismos internacionales, las cuales podrían explicar la recurrencia que se encuentra en el reporte de investigaciones sobre la preferencia estilística de aprendizaje: parece coincidir en que el estilo de mayor frecuencia es el reflexivo-pragmático, lo cual desde el mismo Kolb (1984, 1985) se podría explicar cómo la adaptación del niño a la demanda y necesidad de adaptación al estilo de enseñanza del docente el cual, por lo general es reflexivo-pragmático.

Lo anterior está apoyado por la prueba de hipótesis en la cual no se reportó una diferencia estilística en la preferencia alta/baja por el aprendizaje; esto, podría avalar la idea de que el estilo de aprendizaje más común es el reflejo del estilo de enseñanza del docente. De esta forma, resultaría natural que esa preferencia se desvanezca en los niños al ser testada con una prueba más rigurosa en la cual se pide, bajo cierto nivel de confianza, establecer si esa preferencia existe o no, tal y como se mostró en el presente artículo.

\section{Referencias}

Acevedo, D.; Cavadia, S.; Alvis, A. (2015). Estilos de aprendizaje de los estudiantes de la Facultad de Ingeniería de la Universidad de Cartagena (Colombia). Formación Universitaria, 8(4), 15-22. https://scielo.conicyt.cl/ pdf/formuniv/v8n4/arto3.pdf

Ángel, W.; Alonso, C. (2012). Los estilos de aprendizaje en los estudiantes de primer semestre de la universidad nacional abierta y a distancia Unad, Colombia. Revista de Estilos de Aprendizaje, 5(10), 172-183. http://revistaestilosdeaprendizaje.com/article/view/966/1674

Cáceres, A.; Vilchez, J. (2012). CHAEA junior en estudiantes de la comuna de Talcahuano. En $V$ Congreso Mundial de Estilos de Aprendizaje (pp. 1-10). https://dialnet.unirioja.es/servlet/articulo? codigo $=4675180$

Cánchala, A.; Bastidas, D.; Velásquez, Á. (2019). Estilos de aprendizaje en escolares indígenas del Gran Mallama. Revista UNIMAR, 37(2), 63-71. 
http://editorial.umariana.edu.co/revistas/index.php/unimar/article/ view/2040/2139

Casadiego, J. (2015). Estilos de aprendizaje y rendimiento académico: un estudio con estudiantes de la Facultad de Ingeniería de sistemas de la Universidad Popular del Cesar. [Encuentro Internacional de Educación de Ingeniería, ACOFI]. http://repositorio.upch.edu.pe/bitstream/handle/upch/3848/Estilos_ ColonioGarcia_Luis.pdf? sequence $=1$ \&isAllowed $=\mathrm{y}$

Chiang, M.; Díaz, C.; Arriagada, P. (2016). Estilos de enseñanza y aprendizaje: ¿cómo dialogan en la práctica? Revista de Estilos de Aprendizaje, 9(17), 1-23. http://revistaestilosdeaprendizaje.com/article/view/1045/1758

Coehlo, M.; Amodeo, O.; Gusmão, T.; Muszkat, M. (2013). Analise entre a associação dos estilos de aprendizagem com o gênero, faixa etária e inteligência de crianças brasileiras do ensino fundamental I. Revista de Estilos de Aprendizaje, 6(11), 212-229. http://revistaestilosdeaprendizaje. $\mathrm{com} / \mathrm{article} / \mathrm{view} / 980 / 1688$

Curry, L. (1987). Integrating Concepts of Cognitive or Learning Style: a Review with Attention to Psychometric Standards. Learning Styles Network.

Devalle, A.; Vega, V. (2006). Una escuela en y para la diversidad. El entramado de la diversidad. Aique.

Díaz, D. (2017). Estilos de aprendizaje en estudiantes de educación primaria en el distrito Sapallanga, Huancayo, Perú (Tesis de Maestría). Universidad Nacional del Centro del Perú. http://repositorio.uncp.edu.pe/bitstream/ handle/UNCP/4288/Diaz\%20Medina.pdf? sequence= 1 \&isAllowed $=\mathrm{y}$

Ferrés-Gurt, C.; Marbà-Tallada, A.; Sanmartí-Puig, N. (2014). Trabajos de indagación de los alumnos: instrumentos de evaluación e identificación de dificultades. Revista Eureka sobre Enseñanza y Divulgación de las Ciencias, 12(1), 22-37. https://www.redalyc.org/pdf/920/92032970010.pdf

García, C.; Sáchica, R. (2016). El modelo de aprendizaje experiencial de David Kolb en el aula: una propuesta de intervención y modificación de los estilos de aprendizaje en un grupo de estudiantes de grado cuarto de la I. E. Santa María Goretti de Montenegro, Quindío (Tesis de Maestría). Universidad Católica de Manizales. http://repositorio.ucm.edu.co:8080/jspui/bitstream/ 
handle/10839/1271/Claudia\%20Lorena\%20Garcia\%20Zuluaga. pdf? sequence $=3 \&$ isAllowed $=y$

Gómez, E.; Jaimes, J;; Sereviche, C. (2017). Estilos de aprendizaje en universitarios, modalidad de educación a distancia. Revista Virtual Universidad Católica del Norte, 50, 383-393. https://revistavirtual.ucn.edu.co/index.php/ RevistaUCN/article/view/829

González-Peiteado, M. (2013). Los estilos de enseñanza y aprendizaje como soporte de la actividad docente. Revista de Estilos de Aprendizaje, 1 1(6), 5170. http://revistaestilosdeaprendizaje.com/article/view/971/1679

Granados, L. H.; García, Z. C. (2016). El modelo de aprendizaje experiencial como alternativa para mejorar el proceso de aprendizaje en el aula. Ánfora, 23(41), 37-54. https://www.redalyc.org/pdf/3578/357848839002.pdf

Hederich, C. (2013). Estilística educativa. Revista Colombiana de Educación, 64, 21-56. https://www.redalyc.org/pdf/4136/413634076002.pdf

Hederich, C.; Camargo, Á. (2015). Estilística educativa: un campo de investigación en educación y pedagogía. Revista Latinoamericana de Estudios Educativos, 11(2), 134-167. https://www.redalyc.org/pdf/1341/134146842007.pdf

Hernández, R.; Fernández, C.; Baptista, P. (2014). Metodología de la investigación. McGraw Hill.

Herrera, M. (2014). Estilos cognitivos y estilos de aprendizaje de estudiantes de una institución educativa del sector rural del municipio de Armenia. Universidad de Manizales.

Honey, P; Alonso, C. M. (2002). Cuestionario Honey-Alonso de estilos de aprendizaje: Análisis de sus propiedades Psicométricas en Estudiantes Universitarios. Summa Psicológica UST, 1O(1), 103-117. https://dialnet. unirioja.es/servlet/articulo?codigo $=4421517$

Isaza, L.; Henao, G. (2012). Actitudes-Estilos de enseñanza: su relación con el rendimiento académico. International Journal of Psychological Research, 5(1), 133-141. https://www.redalyc.org/pdf/2990/299023539015.pdf 
Kolb, D. (1984). Experimental Learning: Experience as the Source of Learning and Development. https://www.researchgate.net/publication/235701029_ Experiential_Learning_Experience_As_The_Source_Of_Learning_ And_Development

Kolb, D. (1985). Learning-style Inventory: Self-scoring Inventory and Interpretation Booklet. http://www.sciepub.com/reference/124113

León, C. (2015). La relación de los estilos de aprendizaje y el rendimiento académico en estudiantes de educación primaria (Tesis de Maestría). Tecnológico de Monterrey. https://repositorio.tec.mx/bitstream/ handle/11285/626575/Cielo_Elizabeth_Le\%c3\%b3n_Acosta_. pdf? sequence $=1$ \&isAllowed $=\mathrm{y}$

Lozano, A. (2006). Estilos de aprendizaje y enseñanza. Un panorama de la estilística educativa. Editorial Trillas.

Mejía, M.; Jaik, A. (2014). Estilos de aprendizaje de docentes y alumnos y su relación con el rendimiento académico en educación primaria. Redie Editorial.

Mendoza, W. (2012). Los estilos de aprendizaje en estudiantes universitarios. Estado del arte y desafíos. In $V$ Congreso Mundial de Estilos de Aprendizaje (pp. 1-12). https://dialnet.unirioja.es/servlet/articulo? $\operatorname{codigo}=4665796$

Messick, S. (2010). The Matter of Style: Manifestations of Personality in Cognition, Learning, and Teaching. Journal Educational Psychologist, 29(3), 121-136. https://eric.ed.gov/?id=ED386486

Noriega, N.; Sánchez, A. (2019). Relación entre los estilos de aprendizaje y el proceso de evaluación en la educación primaria. Universidad de la Costa.

Ortiz, E. (2013). La incidencia de los estilos de aprendizaje en el aprendizaje de las matemáticas usando recursos educativos abierto (REA), en estudiantes de $4^{\circ}$ y $5^{\circ}$ de primaria de la escuela rural mercadillo. Universidad Autónoma de Bucaramanga. 
Pantoja, M.; Duque, L.; Meneses, J. (2013). Modelos de estilos de aprendizaje: una actualización para su revisión y análisis. Revista Colombiana de Educación, 64, 79-105. http://www.scielo.org.co/pdf/rcde/n64/n64a04.pdf

Rojas, L.; Zarate, J.; Lozano, A. (2016). La relación entre los estilos de aprendizaje de los estudiantes y los estilos de enseñanza del profesor en un grupo de alumnos de primer semestre del nivel Universitario. Revista de Estilos de Aprendizaje, 9(17), 174-205. http://revista.ieee.es/index.php/ estilosdeaprendizaje/article/view/1051

Santaolalla, E.; Gallego, D.; Urosa, B. (2015). Los libros de texto de matemáticas y su capacidad para desarrollar los distintos estilos de aprendizaje. Revista de Estilos de Aprendizaje, 8(16), 178-210. http:// revistaestilosdeaprendizaje. $\mathrm{com} /$ article/view/1023/1735

Sotillo, J. (2014). El cuestionario CHAEA-Junior o cómo diagnosticar el estilo de aprendizaje en alumnos de primaria y secundaria. Revista de estilos de aprendizaje, 7(13), 182-201. http://revistaestilosdeaprendizaje.com/ article/view/1013/1721

Terrádez, M. (s. f.). Los estilos de aprendizaje aplicados a la enseñanza del español como lengua extranjera. Foro de profesores E/LE, 3, 227-230. https://ojs.uv.es/index.php/foroele/article/view/6525/6312

Uva, A. (2017). Neurociencias en el ámbito educativo. Estilos cognitivos y estrategias de aprendizaje. Revista Internacional Magisterio, 68. https://www.magisterio.com.co/articulo/neurociencias-en-el-ambitoeducativo-estilos-cognitivos-y-estrategias-de-aprendizaje

Varela, M. (2014). Relación entre los estilos de aprendizaje y los niveles de creatividad motriz en los estudiantes de la Institución Educativa las Delicias del municipio de El Bagre. Universidad de Antioquia. 


\section{Anexos}

Anexo 1. Cuestionario CHAEA-Junior de Estilos de Aprendizaje

\begin{tabular}{|c|c|c|}
\hline Más (+) & Menos (-) & Ítem \\
\hline+ & - & $\begin{array}{l}\text { 1. Las personas que me conocen bien consideran que soy fran- } \\
\text { co sobre lo que pienso. }\end{array}$ \\
\hline+ & - & $\begin{array}{l}\text { 2. Para mí es fácil distinguir el bien del mal, lo que está bien y lo } \\
\text { que está mal. }\end{array}$ \\
\hline+ & - & 3. Actúo a menudo sin mirar las consecuencias. \\
\hline+ & - & 4. Me interesa saber cómo piensan los demás y por qué actúan. \\
\hline+ & - & 5. Realmente aprecio los regalos, que son sobre todo prácticos. \\
\hline+ & - & $\begin{array}{l}\text { 6. Trato de ser consciente de lo que está sucediendo donde } \\
\text { estoy. }\end{array}$ \\
\hline+ & - & $\begin{array}{l}\text { 7. Me beneficio si tengo tiempo para preparar mi trabajo y dar } \\
\text { lo mejor de mí. }\end{array}$ \\
\hline+ & - & $\begin{array}{l}\text { 8. Me gusta tener una orden en las comidas, en mis estudios y } \\
\text { hacer ejercicio regularmente. }\end{array}$ \\
\hline+ & - & $\begin{array}{l}\text { 9. Prefiere ideas originales y nuevas, aunque no sean muy } \\
\text { prácticas. }\end{array}$ \\
\hline+ & - & $\begin{array}{l}\text { 10. Acepto y me adapto a los estándares si me permiten lograr } \\
\text { lo que quiero. }\end{array}$ \\
\hline+ & - & 11. Escucho más de lo que hablo. \\
\hline+ & - & $\begin{array}{l}\text { 12. En general, mis cosas en mi habitación son ordenadas, por- } \\
\text { que no puedo soportar el desorden. }\end{array}$ \\
\hline+ & - & $\begin{array}{l}\text { 13. Antes de hacer algo, estudio cuidadosamente sus pros y sus } \\
\text { contras. }\end{array}$ \\
\hline+ & - & $\begin{array}{l}\text { 14. Me interesa más cuando tengo que hacer una actividad } \\
\text { nueva y diferente. }\end{array}$ \\
\hline+ & - & 15. En una discusión me gusta decir claramente lo que pienso. \\
\hline+ & - & $\begin{array}{l}\text { 16. Cuando juego, dejo de lado los sentimientos por mis amigos, } \\
\text { ya que en el juego es importante ganar. }\end{array}$ \\
\hline+ & - & $\begin{array}{l}\text { 17. Me siento cómodo entre personas espontáneas y diverti- } \\
\text { das, aunque a veces me traiga problemas. }\end{array}$ \\
\hline
\end{tabular}




\begin{tabular}{|c|c|c|}
\hline Más (+) & Menos (-) & Ítem \\
\hline+ & - & 18. Expreso abiertamente lo que siento. \\
\hline+ & - & 19. Por lo general, soy el más divertido en las fiestas. \\
\hline+ & - & $\begin{array}{l}\text { 20. Me gusta analizar y devolver ideas para obtener una solu- } \\
\text { ción. }\end{array}$ \\
\hline+ & - & $\begin{array}{l}\text { 21. Prefiero ideas que son útiles para algo y que pueden hacer- } \\
\text { se soñar o soñar despierto. }\end{array}$ \\
\hline+ & - & 22. Tengo cuidado y pienso antes de sacar conclusiones. \\
\hline+ & - & 23. Intento hacer las cosas de manera que sean perfectas. \\
\hline+ & - & $\begin{array}{l}\text { 24. Prefiero escuchar la opinión de los demás antes de exponer } \\
\text { la mía. }\end{array}$ \\
\hline+ & - & $\begin{array}{l}\text { 25. En las discusiones, me gusta observar cómo actúan los } \\
\text { otros participantes. }\end{array}$ \\
\hline+ & - & $\begin{array}{l}\text { 26. No me gusta estar con personas calladas y que cambian } \\
\text { demasiado las ideas. }\end{array}$ \\
\hline+ & - & $\begin{array}{l}\text { 27. Me molesta que tenga que acelerar mi trabajo para comple- } \\
\text { tar un plazo. }\end{array}$ \\
\hline+ & - & 28. Aporto ideas nuevas y espontáneas al trabajo grupal. \\
\hline+ & - & $\begin{array}{l}\text { 29. De vez en cuando, creo que tenemos que saltar los estánda- } \\
\text { res más a menudo de lo que tenemos que obedecerlos. }\end{array}$ \\
\hline+ & - & 30. Cuando estoy con mis amigos hablo más de lo que escucho. \\
\hline+ & - & $\begin{array}{l}\text { 31. Creo que uno siempre debe actuar de manera lógica y } \\
\text { razonada. }\end{array}$ \\
\hline+ & - & 32. Los que dicen cosas sin importancia o locos, me molestan. \\
\hline+ & - & 33. Me gusta comprobar que las cosas realmente funcionan. \\
\hline+ & - & $\begin{array}{l}\text { 34. Rechazo las ideas originales y espontáneas si veo que son } \\
\text { inútiles. }\end{array}$ \\
\hline+ & - & $\begin{array}{l}\text { 35. A menudo reflexiono sobre las consecuencias de mis accio- } \\
\text { nes para planificar el futuro. }\end{array}$ \\
\hline
\end{tabular}




\begin{tabular}{c|c|l|} 
Más(+) & Menos (-) & \multicolumn{1}{|c|}{ Ítem } \\
\hline+ & - & $\begin{array}{l}\text { 36. Muy a menudo, si quieres algo, no importa cómo lo consi- } \\
\text { gas. }\end{array}$ \\
\hline+ & - & $\begin{array}{l}\text { 37. Me molesta cuando un compañero o alguien hace cosas } \\
\text { locamente. }\end{array}$ \\
\hline+ & - & 38. Estoy acostumbrado a pensar sobre temas y problemas. \\
\hline+ & - & $\begin{array}{l}\text { 39. Con frecuencia soy una de las personas que más animan a } \\
\text { las partes. }\end{array}$ \\
\hline+ & - & $\begin{array}{l}\text { 40. Los que me conocen están acostumbrados a pensar que no } \\
\text { soy muy sensible a sus sentimientos. }\end{array}$ \\
\hline+ & - & $\begin{array}{l}\text { 41. Es muy difícil para mí planificar mis tareas y preparar mis } \\
\text { exámenes con el tiempo. }\end{array}$ \\
\hline+ & - & $\begin{array}{l}\text { 42. Cuando estoy en un grupo, es interesante saber lo que } \\
\text { piensan otras personas. }\end{array}$ \\
\hline+ & - & 43. Me molesta que la gente no se tome las cosas en serio. \\
\hline+ & - & $\begin{array}{l}\text { 44. A menudo me doy cuenta de otras formas mejores de hacer } \\
\text { las cosas. }\end{array}$ \\
\hline
\end{tabular}


